\title{
An Enhanced Message Exchange Mechanism in Cluster-Based Mobile Ad Hoc Networks ${ }^{\star, \star \star}$
}

\author{
Wei Lou ${ }^{1}$ and Jie $\mathrm{Wu}^{2}$ \\ 1 Dept. of Computing, Hong Kong Polytechnic University, \\ Hung Hom, Kowloon, Hong Kong \\ 2 Dept. of Computer Science and Engineering, Florida Atlantic University, \\ Boca Raton, Florida, USA
}

\begin{abstract}
In mobile ad hoc networks (MANETs), networks can be partitioned into clusters. Clustering algorithms are localized algorithms that have the property of creating an upper bounded clusterheads in any networks even in the worst case. Generally, clusterheads and selected gateways form a connected dominating set (CDS) of the network. This CDS forms the backbone of the network and can be used for routings (broadcasting/multicasting/unicating). As clusterheads need to determine the selected gateways to connect their adjacent clusterheads within the coverage set, they periodically collect 2-hop and 3-hop clusterhead information. These information can be gathered when they hear their nonclusterhead neighbors sending out messages that contain neighboring clusterhead information. These extra maintenance cost can be reduced when some enhanced mechanism is applied. In this paper, an enhanced mechanism is proposed to reduce the total length of the messages when non-clusterhead nodes exchange their 1-hop and 2-hop neighboring clusterhead information. Simulation shows that over $50 \%$ of message overhead can be saved for dense networks.
\end{abstract}

\section{Introduction}

Mobile ad hoc networks (MANETs) are collections of autonomous mobile hosts without the help of center base stations. Applying such networks into practice brings many challenges to the protocol design, such as routing in highly dynamic networks, allocating shared wireless channels and saving limited bandwidth. Trade-offs are needed in the protocol design to achieve these conflicting goals. One fundamentally problem of MANETs is the scalability issue of the network. As the size of the network increases and the network becomes dense, a flat infrastructure of the network may not work properly, even for a single

* Wei Lou's work was supported in part by the Seed Project Grant of the Department of Computing, Hong Kong Polytechnic University. Contact E-mail: csweilou@comp.polyu.edu.hk

** Jie Wu's work was supported in part by NSF grants CCR 9900646, CCR 0329741, ANI 0073736 and EIA 0130806. Contact E-mail: jie@cse.fau.edu

J. Cao et al. (Eds.): ISPA 2004, LNCS 3358, pp. 223 2322004.

(C) Springer-Verlag Berlin Heidelberg 2004 
broadcast operation [1. Therefore, building some type of hieratical infrastructure for a large network is a necessity and can enhance the performance of the whole network.

The cluster structure is a two-layer hieratical network that converts a dense network to a sparse one, and therefore, relieves the communication overhead of the whole network. The clustering algorithms partition the network into a group of clusters. Each cluster has one clusterhead that dominates all other members in the cluster. Two clusterheads cannot be neighbors. Gateways are those nonclusterhead nodes that have at least one neighbor that belongs to other clusters. It is easy to see that clusterheads and gateways form a connected dominating set (CDS) of the original network. A dominating set (DS) is a subset of nodes such that every node in the graph is either in the set or has an edge linked to a node in the set. If the subgraph induced from a DS of the graph is connected, the DS is a CDS. It has been proved that finding a minimum CDS (MCDS) in a given graph is NP-complete; this applies to a unit disk graph as well [2,3]. In cluster networks, selecting gateways to connect clusterheads and maintaining such a CDS structure in a mobile environment is an extra cost that can be reduced.

Theoretically, we can describe a MANET as a unit disk graph $G=(V, E)$, where the node set $V$ represents a set of wireless mobile hosts and the edge set $E$ represents a set of bi-directional links between the neighboring hosts, assuming all hosts have the same transmission range $r$. Two hosts are considered neighbors if and only if their geographic distance is less than $r . N_{k}(v)$ is $v$ 's $k$-hop neighbor set, including $v$ itself.

When building the infrastructure of the cluster-based CDS in MANETs, each clusterhead collects the clusterhead information within its coverage set so that it can determine some gateways to connect all clusterheads in its coverage set. A node $v$ 's coverage set $C(v)$ is a set of clusterheads that are within a specific coverage area of $v$. It can be a 3-hop coverage set, which includes all the clusterheads in its 3-hop neighbor set $N_{3}(v)$, or a 2.5-hop coverage set, which includes all the clusterheads in $N_{2}(v)$ and the clusterheads that have members in $N_{2}(v)$. These clusterhead information can be gathered by hearing the non-clusterhead neighbors sending out messages that contain neighboring clusterhead information. In this paper, we propose an enhanced mechanism that can reduce the overhead of message exchanges. Instead of sending messages that include all paths to the neighborhood clusterheads, in the enhanced mechanism, each non-clusterhead node sends the message that only provides one path per neighboring clusterhead. In Fig. 1 (a), the original mechanism requires node $g$ to send clusterhead $u$ a message that includes all intermediate nodes (inside the dotted circle) connected to clusterhead $v$. In Fig. 1 (b), the enhanced mechanism requires node $g$ to send clusterhead $u$ a message that only includes an intermediate nodes (grey node) connected to clusterhead $v$. Simulations show that a large amount of overhead can be saved.

The remaining part of the paper is organized as follows: Clustering algorithms are briefly introduced in Section 2. Section 3 describes the message exchanging mechanism. In Section 4 shows the simulation results. Conclusions are drawn in Section 5 . 


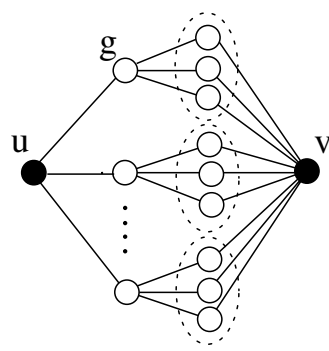

(a)

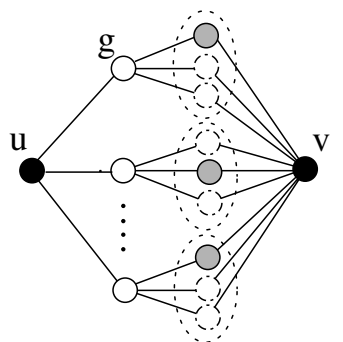

(b)

Fig. 1. Message exchange mechanism: (a) original mechanism and (b) enhanced mechanism

\section{Preliminaries}

The distributed clustering algorithm, lowest-ID clustering algorithm [4, is initiated by electing as a clusterhead the node whose ID is locally the smallest one among all its neighbors. At the beginning, all nodes in the network are candidates. When a candidate finds itself to be the one with the smallest ID among all its 1-hop candidate neighbors, it declares itself as the clusterhead of a new cluster and notifies all its 1-hop neighbors. When a candidate receives a clusterhead notification from a neighboring clusterhead, the candidate joins in the cluster, changes itself to a non-clusterhead member of the cluster, and announces its non-clusterhead state to all its neighbors. If it receives more than one clusterheads' declaration, it joins in the cluster whose clusterhead has the smallest ID. Non-clusterheads that have neighbors belonging to other clusters become gateways. The network will eventually be partitioned into clusters where each cluster has one clusterhead and several gateway/non-clusterhead members. Replacing the clusterhead selecting priority from node ID to effective node degree leads to the highest node degree clustering algorithm 4 .

Jiang et al [5] proposed a cluster-based routing protocol (CBRP) that forms a cluster structure by first electing clusterheads and then letting each clusterhead select one or one pair of gateways to connect to each clusterhead in its adjacent clusters. Therefore, each clusterhead may build multiple paths to its adjacent clusterheads as much as it can maintain.

Kwon and Gerla [6] proposed a passive clustering scheme that constructs the cluster structure during the data propagation. A clusterhead candidate applies the "first declaration wins" rule to become a clusterhead when it successfully transmits a packet. Then, its neighbor nodes can learn the presence of this clusterhead and change their states to become gateways if they have more than one adjacent clusterhead or ordinary (non-clusterhead) nodes otherwise. The passive clustering algorithm has the advantages of no initial clustering phase, no need of the complete neighborhood information for the clusterhead election and no communication overhead for maintaining cluster structure or updating neighborhood information, but it suffers poor delivery rate and global parameter requirement. 
Alzoubi et al 7] proposed a cluster-based message-optimal CDS which is formed with two steps: In the first step, clusterheads are determined by the lowest-ID clustering algorithm. A clusterhead knows all its 2-hop and 3-hop clusterheads with two rounds of neighborhood information exchanges. In the second step, each clusterhead selects a node to connect each 2-hop clusterhead and a pair of nodes to connect each 3-hop clusterhead. All the clusterheads and selected nodes form a CDS of the network.

Lou and $\mathrm{Wu} 8$ proposed two categories of cluster-based CDSs. Both clusterbased CDSs can be built on the 3-hop or 2.5-hop coverage set. These algorithms are localized algorithms since each clusterhead only selects some gateways to connect the clusterheads within the coverage set. In general, the size of a clusterhead's 2.5 hop coverage set is less than that of its 3 hop coverage set. Therefore, the cost of maintaining the 2.5-hop coverage set is less than that of the 3-hop coverage set.

One common feature of all the above algorithms is that each clusterhead needs two rounds of neighborhood information exchanges and overhead of these neighborhood information is not taken into consideration when the clustering algorithm is designed.

\section{An Enhanced Mechanism of Exchanging Neighborhood Information}

Constructing clusters in a MANET needs several rounds of message exchanges. Also, to build the cluster-based CDS of the network, each clusterhead has to gather neighboring clusterhead information within its coverage set. These information comes from the neighboring clusterhead messages sent by those nonclusterheads.

\subsection{Construction Process}

The construction of the cluster-based backbone is described in detail as follows:

At the beginning, each node can learn its neighbors' IDs through HELLO messages. The network is partitioned into clusters by applying the lowest-ID clustering algorithm. A clusterhead will send out a $\mathrm{CH}$ message and a nonclusterhead will send out a NCH message to inform its neighbors.

After the clusters have been formed, each node knows all its 1-hop neighbors. A non-clusterhead $v$ sends out a 1-hop neighboring clusterhead message CH_HOP1 $(v)$ which includes all $v$ 's 1-hop neighboring clusterheads. When the clusterhead $u$ receives the CH_HOP1 messages from all its non-clusterhead neighbors, $u$ adds an entry for each new neighboring clusterhead with its associated gateway that connects the clusterheads together.

Once another non-clusterhead $w$ receives the message $\mathrm{CH} \_\mathrm{HOP} 1(v)$ from $v$, $w$ builds the message CH_HOP2 $(w)$ as follows: If a clusterhead $u$ that is found in $\mathrm{CH} \_\mathrm{HOP} 1(v)$ is also a 1-hop neighbor of $w, w$ ignores $u$ in the CH_HOP2 $(w)$; Otherwise, $w$ checks if $u$ is a new 2-hop clusterhead of $w$. If so, $w$ creates a new entry that contains the 2-hop clusterhead $u$ and the its associated node $v$. If $u$ 
is already included in the entry and the associated node $v$ has a higher priority (e.g., lower ID or higher linkage quality, etc.) than the original one, the entry can be updated with the new associated node $v$.

When $w$ receives the CH_HOP1 messages from all its non-clusterhead neighbors, it sends out a message CH_HOP2 $(w)$ that contains all 2-hop clusterhead entries. Unlike other algorithms that each clusterhead includes all associated gateways in the message $\mathrm{CH}$ _HOP2, the $\mathrm{CH}$ HOP2 with enhanced mechanism builds each entry with one clusterhead and one associated gateway. Therefore, the size of the message CH_HOP2 can be much smaller. When the clusterhead $u$ receives a CH_HOP2, $u$ builds a new entry for each clusterhead in the CH_HOP2.

After clusterhead $u$ receives all CH_HOP1 and CH_HOP2 messages from its non-clusterhead neighbors, $u$ builds its coverage set $C(u)=C_{2}(u) \cup C_{3}(u)$, where $C_{2}(u)$ consists of all elements in CH_HOP1 and $C_{3}(u)$ consists of all elements in CH_HOP2. If a clusterhead appears in both $C_{2}(u)$ and $C_{3}(u)$, the one in $C_{3}(u)$ is removed.

Each clusterhead $u$ selects gateways to connect all the clusterheads in $C(u)$ with the forward node set selection process. The gateways are selected by the greedy algorithm: The neighbor node or the pair of nodes, whichever has the highest "yield" is first selected as the gateway(s). A "yield" of node(s) is defined as the total number of the clusterheads in the $C(u)$ that was connected by the selected gateway(s) divided by the number of the selected gateway(s). A tie of yield is broken by selecting the smaller node ID. When a node is selected, all of the clusterheads in $C(u)$ is removed. The selection process repeats until $C(u)$ is empty.

After a clusterhead determines its gateways, it sends out a GATEWAY message that contains all selected gateways. The selected 1-hop gateways forward the GATEWAY message so that all the selected 2-hop gateways can be informed.

\section{$3.2 \quad$ Example}

Fig. 2 shows the construction process of a cluster-based CDS backbone. At the beginning, all the nodes are candidates (Fig. 2(a)). With the lowest-ID clustering (LID) algorithm, nodes 1, 2, 4, 8 and 10 become clusterheads and form clusters labelled as $C_{1}, C_{2}, C_{3}, C_{4}$ and $C_{5}$; then nodes 7 and 9 join in cluster $C_{1}$, nodes 3 and 6 join in cluster $C_{2}$,nodes 5 joins in cluster $C_{3}$ (Fig. 2 (b)).

Fig. 2(c) illustrates message exchange when node 4 construct its 3-hop and 2.5 hop coverage sets:

(1) For 3-hop coverage set, node 6 sends CH_HOP1(6) (M1 in Fig. 2(c)) which contains its 1-hop clusterhead neighbor set $\{2 *\}, 3$ sends CH_HOP1(3) (M2 in Fig. 2(c)) contains $\{2 *, 8\}$. Here, $*$ indicates the clusterhead of the cluster that the node belongs to. Likewise, nodes 5 and 7 send $\mathrm{CH} \_\mathrm{HOP} 1(5)=\{4 *, 10\}$ and $\mathrm{CH} \_\mathrm{HOP} 1(7)=\{1 *, 4\}$ (M3 and M5 in Fig. 2(c)). After receiving M1 and M2, node 7 may form CH_HOP2(7) (M6 in Fig. 2(c)) which contains its 2-hop clusterhead neighbors and associated gateways $\{2[3], 8[3]\}$. Here, CH_HOP2 $(u)=$ $\{v[w], \ldots\}$ means that clusterhead $u$ connects to clusterhead $v$ via $w$. Then node 7 sends CH_HOP2(7) out. Note that node 7 picks node 3 as the gateway instead 


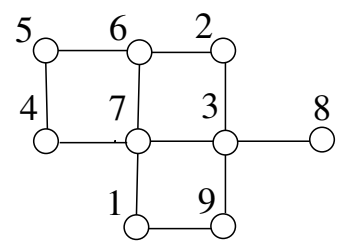

(a)

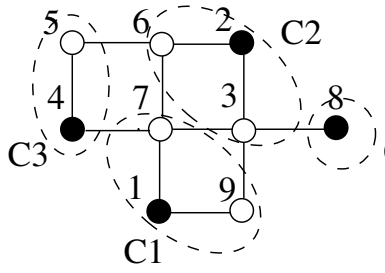

(b)

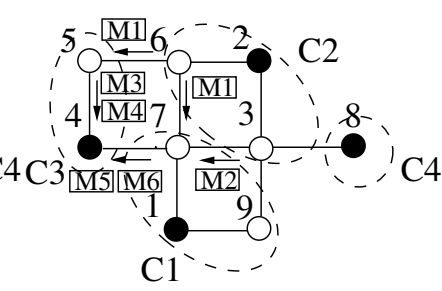

(c)

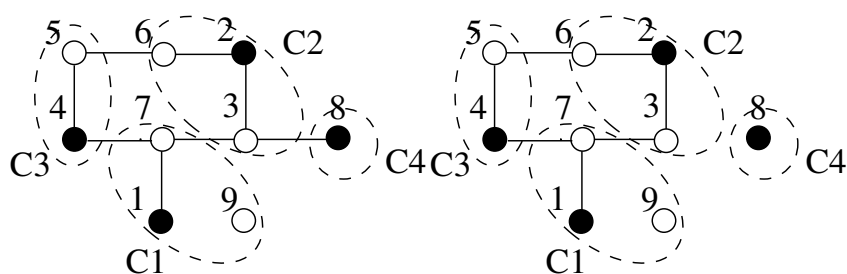

(d)

(e)

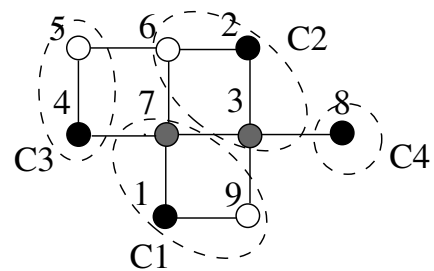

(f)

Fig. 2. Illustration of constructing a coverage set: (a) initial network, (b) clusters, (c) exchange neighboring information, (d) 3-hop coverage set, (e) 2.5-hop coverage set, and (f) cluster-based CDS

of node 6 because node 3 has two adjacent clusterheads 2 and 8 . Also, the size of the CH_HOP2(7) is smaller than the one that includes all possible paths to node 2 since it only keeps the most favorable path in the message. Similarly, node 5 will send CH_HOP2 $(5)=\{2[6]\}$ out (M4 in Fig. 2(c)). After receiving M3, M4, M5 and M6, node 4 can build its local view of its coverage set (Fig. 2(d)). 
Clusterhead 4 selects node 5 as the gateway to connect to clusterhead 10 , selects nodes 7 and 3 as the gateways to connect clusterheads 1, 2 and 8, and sends a message GATEWAY $(1)=\{3,5,7\}$. Similarly, clusterheads 1 and 8 select nodes 7 and 3 as gateways and send $\operatorname{GATEWAY}(1)=\operatorname{GATEWAY}(8)=\{3,7\}$; clusterhead 2 selects node 3 to connects clusterhead 8 , selects nodes 3 and 7 to connect clusterheads 1 and 4 , selects nodes 6 and 5 to connect clusterhead 10 , and sends GATEWAY $(2)=\{3,5,6,7\}$; clusterhead 10 selects nodes 5 and 6 to connect clusterheads 2 and 4 , and sends $\operatorname{GATEWAY}(10)=\{5,6\}$. The final cluster-based CDS is $\{1,2,3,4,5,6,7,8,10\}$ (Fig. 2 (f)).

(2) For the 2.5-hop coverage set, node 4 builds its local view as Fig. 2(e). Here, clusterhead 4 does not know clusterhead 8 since CH_HOP2(7) only includes $\{2[3]\}$. Therefore, the size of the CH_HOP2(7) is even reduced. The forward node set selection process is similar and the final cluster-based CDS is $\{1,2,3,4,5,6,7,8,10\}$ (Fig. 2 (f)).

\subsection{Message Overhead Complexity}

The message exchange overhead for constructing a cluster-based CDS of the network is listed in Table 1, Note that the number of clusterheads within 1-hop and 2-hop neighbor set are bounded by a constant value [7], the size of CH_HOP1 and $\mathrm{CH} \_$HOP2 with the enhanced message exchange mechanism are $\mathrm{O}(1)$. On contrast, the original one without this mechanism will send message CH_HOP2 that containing all possible gateways. Therefore, the size of $\mathrm{CH}_{-} \mathrm{HOP} 2$ is $\mathrm{O}(\Delta)$, where $\Delta$ is the maximum node degree of the network.

Table 1. Message Overhead Complexity

\begin{tabular}{|c|c|c|}
\hline \multirow[b]{2}{*}{ Message type } & \multicolumn{2}{|c|}{ Algorithm } \\
\hline & Original & Enhanced \\
\hline HELLO & $\overline{\mathrm{O}(1)}$ & $\overline{\mathrm{O}(1)}$ \\
\hline $\mathrm{CH}(\mathrm{NCH})$ & $\mathrm{O}(1)$ & $\mathrm{O}(1)$ \\
\hline CH_HOP1 & $\mathrm{O}(1)$ & $\mathrm{O}(1)$ \\
\hline CH_HOP2 & $\mathrm{O}(\Delta)$ & $\mathrm{O}(1)$ \\
\hline GATEWAY & $\mathrm{O}(1)$ & $\mathrm{O}(1)$ \\
\hline Total & $\mathrm{O}(\Delta)$ & $\mathrm{O}(1)$ \\
\hline
\end{tabular}

\section{Simulations}

We measure the average sizes of the message exchange for constructing the cluster-based CDS with enhanced message exchange mechanism (referred to as Enhanced (3-hop and 2.5-hop) and without enhanced message exchange mechanism (referred to as Original (3-hop)). The size of the message is counted as the number of nodes included in the message.

The simulation runs under the following simulation environment: A number of nodes (ranging from 100 to 1000) are randomly placed in a confined working 
space $100 \times 100$. The nodes have the same transmission ranges, and the link between two nodes is bi-directional. The network is generated with two fixed average node degrees: $d=6$ and 18, which are the representatives of the relative sparse and dense networks. If the generated network is not connected, it is discarded. We only consider the traffic of the packets at the network layer without any transmission collision. We repeat the simulation until the $99 \%$ confidential interval of the result is within $\pm 5 \%$.

Table 2. Message exchange overhead (sparse network: $n=1000, d=6$ )

\begin{tabular}{||c|c|c|c||}
\hline \multirow{2}{*}{ Message } & \multicolumn{3}{|c||}{ Algorithm } \\
\cline { 2 - 4 } & Original(3-hop) & Enhanced(3-hop) & Enhanced(2.5-hop) \\
\hline \hline HELLO & 1000.0 & 1000.0 & 1000.0 \\
\hline CH(NCH) & 1000.0 & 1000.0 & 1000.0 \\
\hline CH_HOP1 & 1209.3 & 1209.3 & 1209.3 \\
\hline CH_HOP2 & 5106.3 & 3874.7 & 2973.7 \\
\hline GATEWAY & 1134.0 & 1134.0 & 1013.3 \\
\hline \hline Total & 9449.6 & 8218.0 & 7197.3 \\
\hline
\end{tabular}

Table 3. Message exchange overhead (dense network: $n=1000, d=18$ )

\begin{tabular}{||c|c|c|c||}
\hline \multirow{2}{*}{ Message } & \multicolumn{3}{|c||}{ Algorithm } \\
\cline { 2 - 4 } & Original(3-hop) & Enhanced(3-hop) & Enhanced(2.5-hop) \\
\hline \hline HELLO & 1000.0 & 1000.0 & 1000.0 \\
\hline CH(NCH) & 1000.0 & 1000.0 & 1000.0 \\
\hline CH_HOP1 & 1601.8 & 1601.8 & 1601.8 \\
\hline CH_HOP2 & 15587.6 & 6912.5 & 5421.9 \\
\hline GATEWAY & 837.7 & 837.7 & 724.4 \\
\hline \hline Total & 20027.1 & 11352.0 & 9748.1 \\
\hline
\end{tabular}

The Tables 2 and 3 show the cases that the sizes of different types of messages exchanged for the construction of the clusters of the network, when the number of nodes in the network is 1000 and the average node degree is 6 and 18 respectively. We can see that the size of CH_HOP2 is the most weighted part of the total sizes $(40 \% \sim 70 \%)$. In the sparse scenario where average node degree $d$ is 6 , the enhanced mechanism can reduce $25 \%$ of overhead of CH_HOP2 with 3-hop coverage set and $17 \%$ less with 2.5-hop coverage set compared with the original one. The overhead of the total size of the message can reduce $13 \%$ and $24 \%$ of the original one with 3-hop and 2.5-hop coverage set. In the dense network where average node degree $d$ is 18 , the reduction of the overhead of the CH_HOP2 can reach $56 \%$ with 3 -hop coverage set and $66 \%$ with 2.5 -hop coverage. Correspondingly, the total size of the message can reduce $43 \%$ and $51 \%$ of the original one.

The Fig. 3 shows the average size of message per node when the size of the network ranges from 100 to 1000. Fig. 3 (a) shows the scenario when the 

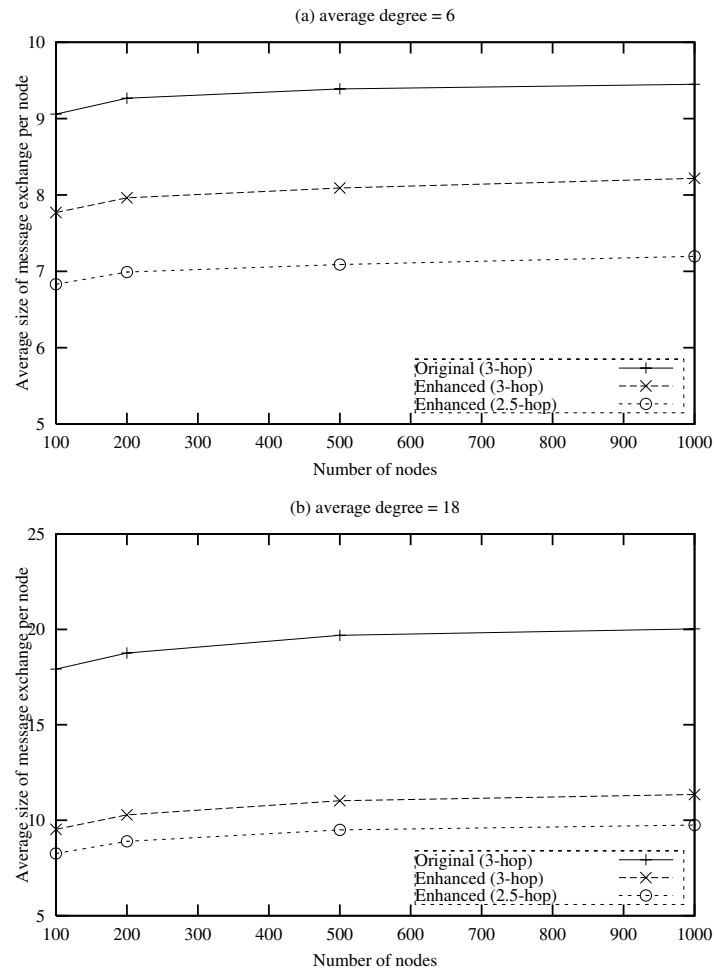

Fig. 3. Message Overhead: (a) average node degree $=6$, and (b) average node degree $=18$

node degree is 6 . The cost decreases from over 9 for the original one to around 8 (over 10\%) for the enhanced mechanism with 3-hop coverage set, and then to around 7 (over 20\%) for the enhanced mechanism with 2.5-hop coverage set. Fig. 3 (b) shows the scenario when $d$ is 18. The cost decreases more remarkably from near 20 for the original one to around 10 (50\%) for the enhanced mechanism with 3-hop coverage set and even less for that with 2.5hop coverage set. We observe that overhead total cost of message exchange per node increases remarkably (doubled) when the network becomes dense. Also, we find that the enhanced mechanism can greatly reduce the message exchange overhead, especially for the dense network. The reason is that when the network is dense and each node has more neighbors, a CH_HOP2 will include more different intermediate nodes for each 2-hop clusterhead for the original mechanism. Thus, the overhead of CH_HOP2 increases remarkably. When the CH_HOP2 includes only one intermediate node for each 2-hop clusterhead for the enhanced mechanism, the message overhead can be greatly reduced. 


\section{Conclusions}

In this paper, an enhanced message exchange mechanism is proposed to reduce the size of the messages when the network forms into clusters. When the nonclusterhead nodes only exchange their 2-hop neighboring clusterhead with only one intermediate node, the message complexity can be reduced from $\mathrm{O}(\Delta)$ to $\mathrm{O}(1)$. Simulation shows that this mechanism can greatly reduce the total message overhead. When the network is dense network, over $50 \%$ of message overhead can be saved.

\section{References}

1. S. Ni, Y. Tseng, Y. Chen, and J. Sheu: The broadcast storm problem in a mobile ad hoc network. Proc. of ACM/IEEE MOBICOM'1999 (1999), 151-162.

2. V. Chvatal: A greedy heuristic for the set-covering problem. Mathematics of Operation Research, 4(3) (1979), 233-235.

3. M. V. Marathe, H. Breu, H. B. Hunt III, S. S. Ravi, and D. J Rosenkrantz: Simple heuristics for unit disk graphs. Networks, 25 (1995), 59-68.

4. A. Ephremides, J. E. Wieselthier, and D. J. Baker: A design concept for reliable mobile radio networks with frequency hopping signaling. Proc. of the IEEE, 75(1) (1987), 56-73.

5. M.L. Jiang, J. Y. Li, and Y. C. Tay: Cluster based routing protocol (CBRP) functional specification. IETF Internet draft (1999), http://www.ietf.org/ietf/draft-ietfmanet-cbrp-spec-01.txt.

6. T. J. Kwon and M. Gerla: Efficient flooding with passive clustering (PC) in ad hoc networks. ACM Computer Communication Review, 32(1) (2002), 44-56.

7. K. M. Alzoubi, P. J. Wan, and O. Frieder: Message-optimal connected dominating sets in mobile ad hoc networks. Proc. of ACM/IEEE MOBIHOC'2002 (2002), 157164 .

8. W. Lou and J. Wu: A cluster-based backbone infrastructure for broadcasting in manets. Proc. of IEEE IPDPS'2003, Workshop of WMAN (2003). 